\title{
Jumping technique and climbing behaviour of the collembolan Ceratophysella sigillata (Collembola: Hypogastruridae)
}

\author{
Jürg ZETTEL, URsula ZETTEL and BeAtrice EGGER \\ Zoological Institute, University of Bern, Baltzerstr. 3, CH-3012 Bern, Switzerland; e-mail: juerg.zettel@zos.unibe.ch
}

Key words. Collembola, Hypogastruridae, Ceratophysella sigillata, jumping, orientation, climbing behaviour, migration, eversible vesicles, polymorphism

\begin{abstract}
Ceratophysella sigillata uses protrusible vesicles on its antennae and posterior end as sticky landing devices to avoid tumbling upon landing. This technique facilitates its orientated movement during winter migration. It allows the animal to use directional jumps without rebounding even when climbing tree trunks. The antennal vesicles are present only in the surface active morphs of $C$. sigillata, and only these individuals jump readily. The vesicles are everted only when the animal has time to prepare the jump. In an escape leap, they are not protruded and therefore the animal tumbles upon landing.
\end{abstract}

\section{INTRODUCTION}

In Collembola, the usual way to move is by crawling but jumping is used as a very efficient mechanism to escape from attack or disturbance. Details on this extremely rapid movement have been given by Bauer \& Völlenkle (1976) and Christian (1978, 1979). Jumps are energy-consuming and after a few repeated leaps the individual springtail has used its available ATP and remains immobilized until the energy reserves have been regenerated (Ruhfus \& Zinkler, 1995; personal observations).

Winter active Collembola jump regularly when migrating. This behaviour has been described for Hypogastrura socialis (Lyford, 1975; Leinaas, 1981a; Hågvar, 1995), for $H$. lapponica (Leinaas, 1981b), and we have found it in all the species we observed during winter activity so far: Hypogastrura socialis, Ceratophysella sigillata, Vert agopus westerlundi, Isotoma hiemalis, Isotomurus schaefferi, Agrenia bidenticulata.

By strictly orientated jumping, Collembola are able to cover remarkable distances within a short time; in C. sigillata we observed up to $3 \mathrm{~m}$ per h, and Hågvar (1995) calculated up to 200-300 m per day for $H$. socialis.

Several authors report on the ability of Collembola for visual orientation (Müller-Kraenner, 1990; Hågvar, 1995, own observations)

As springtails rotate during their leaps and land at high speed, they tumble upon landing and it takes them some time before being able to move on in a specific direction.

The present paper describes the jumping behaviour in Ceratophysella sigillata, which is able to avoid tumbling by a special technique, being not only of significance during migration, but also for feeding behaviour.

\section{MATERIAL AND METHODS}

Ceratophysella sigillata (Uzel) is a winter active hypogastrurid known from the northern forelands of the Alps (Palissa, 1994), but also from North America: Babenko et al. (1964) established C. pseudoarmata (Folsom, 1916) to be a junior synonym of $C$. sigillata. First observations on its biology were made by Zettel \& Zettel (1994a, b). The collembolan shows an unusual migration behaviour during winter, with dense colonies comprising up to hundreds of millions of individuals moving over the surface in the same direction.

\section{Field observations}

Since 1990 we have investigated this species in a mixed forest $10 \mathrm{~km}$ north of Berne, at an altitude of $640 \mathrm{~m}$ a.s.l. The present data are part of a programme covering the migration patterns of this species. Individual jumping behaviour was observed either with a short focus telescope (magnification $\times 8$; on the soil surface, at a distance of 1.5 to $2 \mathrm{~m}$ ) or with a dissecting microscope (magnification $\times 14$; climbing on trees, at a distance of ca. 20 $\mathrm{cm}$ ), both instruments being mounted on a tripod. No artificial illumination was necessary for both types of observation and the animals did not seem to be influenced by the presence of the observers.

\section{Observations in the laboratory}

Some aspects of the behaviour had to be investigated in the laboratory, using a dissecting microscope with a magnification up to $\times 40$. The animals were kept in climatic chambers and observed directly in the culture vessels (glass vials of a diameter of $8 \mathrm{~cm}$ with a moist base layer of plaster of Paris).

Observations concerning orientation and jumping distance were carried out in a climatic chamber at field temperature $\left(0-5^{\circ} \mathrm{C}\right)$. Individuals were released from a culture vessel onto a big sheet of rough paper and their positions were recorded directly by tracing their displacements with a pencil. The marking of starting and landing points as well as positions at intervals of 10 seconds during crawling could be done at a distance of $1 \mathrm{~cm}$ behind the animals without disturbing them. To exclude an orientation on landmarks, the animals had to move in an arena surrounded by black paper. The light source was positioned either on one side of the arena or directly above it, producing a diffuse illumination against the ceiling.

\section{Photography in the laboratory}

To analyze the sequences of takeoff and landing, we took photographs with a Leitz Photomakroskop (magnification up to $\times 32)$ and a high speed flash $(1 / 8000 \mathrm{sec})$. All pictures were taken in a climatic chamber at the actual culture temperature of $5-10^{\circ} \mathrm{C}$. 
It proved much easier to take several single shots to obtain pictures of all positions in the sequence than to use a high resolution stroboscopic flash or an ultraspeed film camera, because we needed pictures of undisturbed and spontaneously jumping animals. The take-off was recorded from the tip of a short vertical glass capillary into which some animals had been dropped; they climbed up and when emerging at the tip of the capillary, many of them prepared to jump. By illuminating from one side, they could be guided in the desired direction. Landing was either recorded frontally through a vertically mounted microscope cover slide, against which the jumps were directed by the lighting, or from an angle of ca. $45^{\circ}$ from above in the culture vessels. Shutter and flash were triggered manually when an animal appeared within a certain frame.

Among ca. 200 pictures taken, one third was successful, i.e. provided photographs of all positions needed for the interpretation of the extremely rapid movements during take-off and landing. During the preparation for the jump it was easy to trigger the shutter at the appropriate moment, because this sequence lasted at least $0.5 \mathrm{~s}$. To record the landing, several hundred individuals could be made to simultaneously start to climb the glass wall of the culture vessel, orientating towards the light source outside the jar. By this method, we often photographed several different positions among $30-50$ animals in the focus plane.

\section{RESULTS}

\section{Jumping morphs}

C. sigillata passes through 4 morphs within one year of its life (for details see Zettel \& Zettel, 1994b), but only two of them are surface active. One of these can be observed in December/January and February/March, the other in April/May. During favourable humidity conditions, the winter active morph climbs onto trees to feed on coccal algae growing on the trunks.

Only the two surface active morphs jump readily, the other two which remain in the litter or soil show jumping only as an escape behaviour. As a typical hypogastrurid, C. sigillata has eversible anal vesicles. An additional and important feature of the surface active morphs is an eversible vesicle between the antennal segments 3 and 4 (being a characteristic of the genus Ceratophysella), which is lacking in the soil dwelling morphs. These eversible parts proved to be of central significance in the jumping sequence.

\section{Orientation and jumping frequency}

Figs 1 and 2 show that a light source can be used for orientation. With a distinct light source, most animals moved in a strongly directional way which was not necessarily orientated towards the light source as it was the case in Fig. 1; the majority of the animals used in an experiment moved in the same direction. Under a diffuse illumination no linear trajectories could be observed. With a visible light source, jumping was significantly more frequent than under diffuse lighting when $C$. sigillata showed no orientation ( $P<0.001, \chi^{2}$ test). A mean jump measured $54 \pm 0.5 \mathrm{~mm}$ (mean $\pm 95 \%$ C.I.) and the leaps tended to be shorter without an orientation possibility.

As the Collembola do not land on their feet, they had to grip the substratum and reorientate after landing. They orientated by turning on the spot until their body axis was in the same direction as the preceding jumps, sometimes combined with some short crawling. In the field a significant difference between the reorientation times in colonies being mainly resident or during migrating was observed. In moving colonies the mean time between two jumps varied between $10.1 \pm 1.06$ and $11.1 \pm 2.1 \mathrm{~s}(\mathrm{n}=$ 50 ; mean $\pm 95 \%$ C.I.). In resident aggregations the time was between $16.9 \pm 1.80$ and $18.9 \pm 2.37 \mathrm{~s}$. Animals not aggregated in dense colonies spent more time crawling, two leaps being separated by $91.5 \pm 71.95 \mathrm{~s}(\mathrm{n}=8)(\mathrm{Eg}-$ ger, 1998).

\section{Preparation for jumping}

There is a clear difference between a spontaneous takeoff (when migrating) or when disturbed. Whenever possible the animals prepare for a jump, this lasts at least 0.5 seconds. Three steps can be distinguished during this phase (Figs 3,4). As soon as an animal has decided in which direction to jump, it takes a firm grip with its feet on the substratum and contracts its body muscles, resulting in a visible shortening of the body. By increased tur-
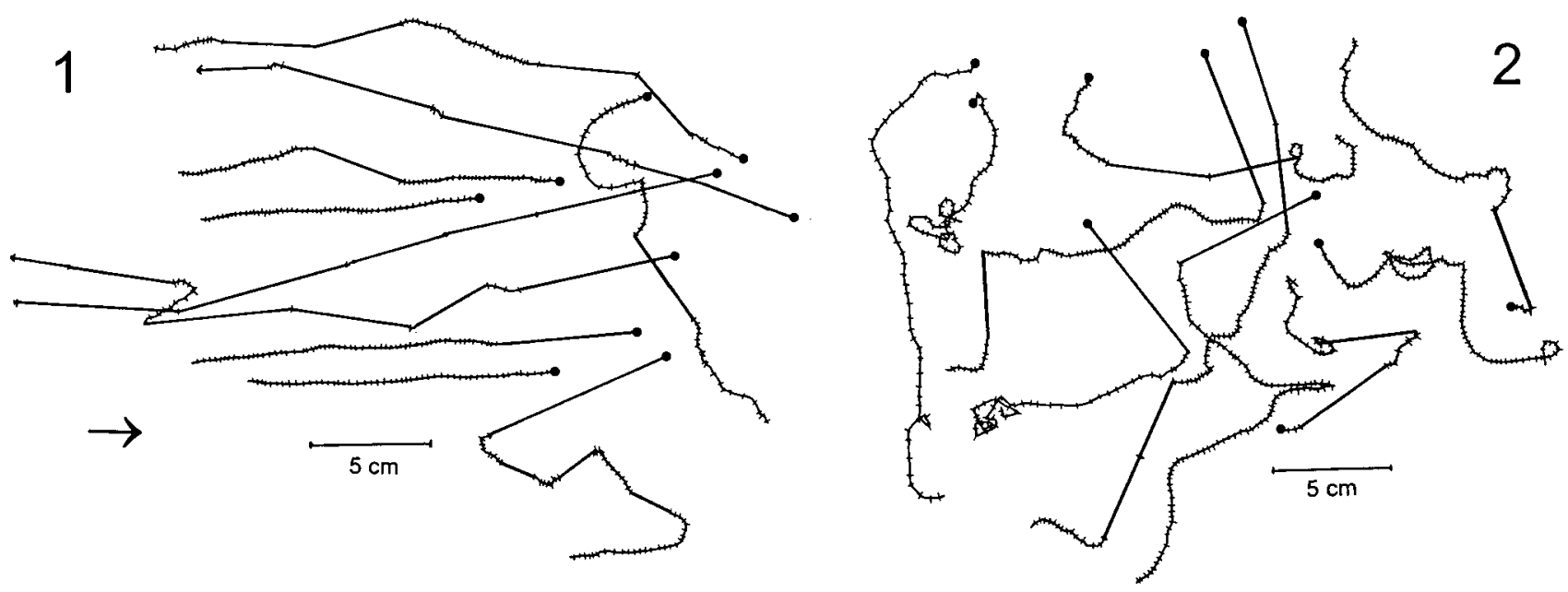

Figs 1,2 . Trajectories of migrating $C$. sigillata in a climatic chamber at $5^{\circ} \mathrm{C}$. Positions are marked at intervals of $10 \mathrm{~s}$ and jumps displayed with bold lines. 1 - strictly directional movements with frequent jumping under direct illumination from one side (arrow); 2 - disorientated movements with reduced jumping under diffuse light conditions. 


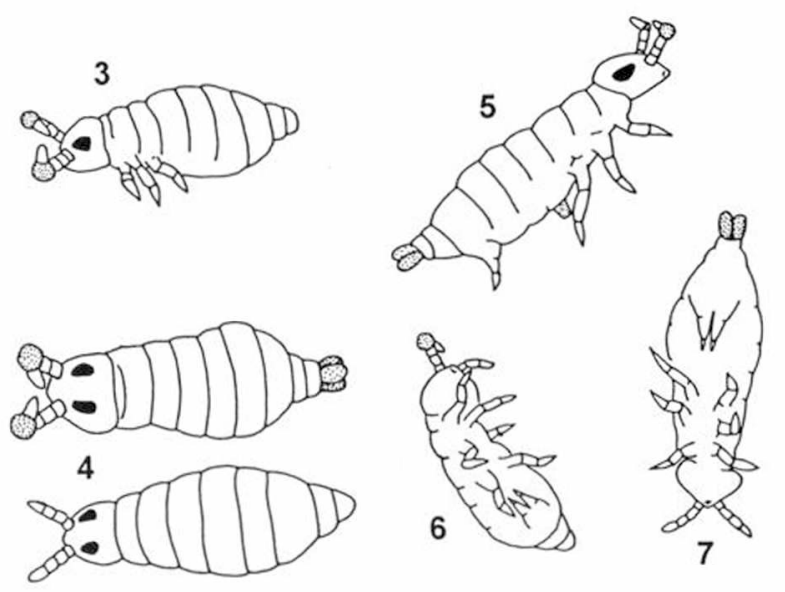

Figs 3-7. All figures are drawn from microphotographs. Everted parts (vesicles, ventral tube) are stippled. 3 - preparation for takeoff in C. sigillata: the antennal vesicles are fully protruded, the abdomen is already contracted, but the anal vesicles are not visible; 4 - comparison of the normal attitude of $C$. sigillata with an individual ready to jump, all vesicles protruded; 5 - an individual of $C$. sigillata in full flight, immediately before hitting a vertical surface: due to the high internal body pressure, all eversible vesicles and the ventral tube are protruded, legs and furca fully spread; $6-C$. sigillata after landing head first. The animal sticks to the glass wall with its right antennal vesicle, moving with its legs. Internal body pressure is already normal, anal vesicles withdrawn and furca flexed; $7-C$. sigillata after hitting the glass wall with its posterior end first: it hangs on the anal vesicles, moving with its legs, antennal vesicles already withdrawn and furca flexed. gor the eversible antennal vesicles are protruded, and the tips of the antennae bent upwards and inwards. In the final step the internal pressure is further increased and the anal vesicles as well as the ventral tube are everted. These parts remain protruded during the entire leap (Fig. 5). When disturbed, the animals are able to take off without preparation within milliseconds, but in this case neither antennal nor anal vesicles are protruded.

\section{Landing}

The difference between these two jumping behaviours becomes obvious upon landing. After an escape leap, the landing is the same as after any collembolan jump: due to the enormously high speed, the animal is tumbling and has to struggle to get onto its feet again. After a prepared leap, the animal sticks to the substratum and does not tumble. Due to their wet surface, the protruded vesicles act as sticking devices. A landing collembolan can hit the ground directly either with its antennae or its posterior end (Figs 6,7); in such cases it sticks to the surface and by bending its body and moving its legs it regains its feet and continues directional movement. When landing on its ventral or dorsal side, the body continues to rotate in the direction of the leap and there is immediately contact between the sticky vesicles and the surface. On a horizontal surface (cardboard exposed in the field) $30 \%$ of the landing animals became stuck with the antennae, $20 \%$ with the anal vesicles, $50 \%$ landed with another part of the body first. On a vertical obstacle placed in the way of a

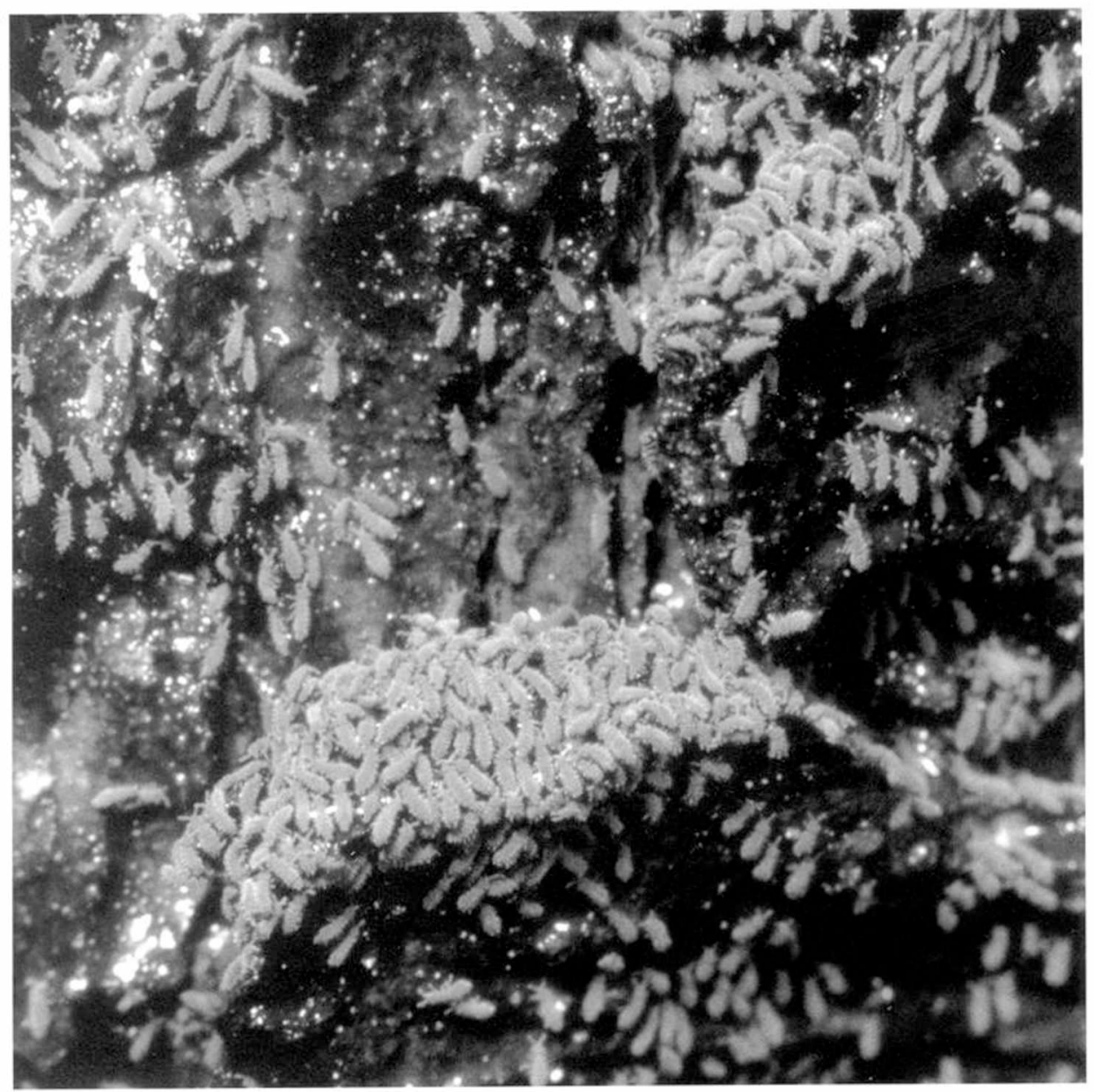

Fig. 8. C. sigillata crowding on the tip of a bark flake when climbing a spruce trunk. 
migrating colony, $60 \%$ landed head first and stuck with their antennal vesicles, $40 \%$ stuck with their anal vesicles (Egger, 1998).

\section{Jumping during climbing}

During surface activity and when the tree trunks are moist, $C$. sigillata often climbs to several metres in height, to feed on coccal algae growing on the trunks (Suter, 1993). There is a difference when climbing a beech or a spruce trunk. The bark of beech (Fagus sylvatica) is smooth and the climbing springtails move continuously upwards without being hindered by obstacles. The trunk of spruce (Picea abies) is covered by projecting bark flakes. Each flake is a dead end for climbing Collembola, which leads to crowding at the edge of each flake as the animals attempt to escape from it (Fig. 8). When the crevice behind the flake is deeper than a few millimetres, a few individuals climb down on the rear side of the flake, but most jump towards the bark behind the flake, where they stick with their (antennal) vesicles without rebound and continue to climb upwards until the next flake is reached (Egger, 1998).

\section{DISCUSSION}

\section{Jumping in Collembola}

Up to now investigations of springtail jumping have been carried out by individuals being forced to jump by a mechanical or chemical stimulus. In such cases the jump can be predicted precisely - allowing the use of high speed cinematography. In undisturbed individuals, takeoff may occur anytime within several seconds after the first signs of preparation. Therefore we chose an alternative recording technique to analyze the preparation for a jump as well as the take-off and landing.

From high speed cinematographic pictures (Christian, 1979; Brackenbury \& Hunt, 1993) and the models by Brackenbury \& Hunt (1993) it can be seen that animals with a long furca perform steep and often backward orientated somersaults, but species with a short furca like the hypogastrurids show relatively low trajectories with a much slower rotation.

It is interesting to realise that only the surface active morphs of $C$. sigillata jump spontaneously and only these individuals have antennal vesicles. The morphs remaining in the soil lack antennal vesicles and do not jump spontaneously.

\section{Orientation}

The laboratory experiments showed that $C$. sigillata is able to orientate with the aid of a light source. The chosen direction was not obligatorily towards the light and could chance with time. However, orientation during migration cannot be based only on such a mechanism, otherwise the animals would change their direction continuously during the day according to the sun. This was not the case, migrating colonies often kept their direction for several days. Nothing is known about how springtails are able to compensate for the movement of the sun, as is known for some insects. Observations by Hågvar (1995) suggest that coarse patterns in the environment can be used for orien- tation, and that a directional movement is also possible without sun. We never observed a deorientation of $C$. sigillata under an overcast sky. It is possible that $C$. sigillata is able to use other cues in addition to visible light.

Müller-Kraenner (1990) showed that light-dark patterns were used for visual orientation in Entomobrya corticalis to enable movement towards tree trunks. Our observations in the forest indicated that $C$. sigillata was able to perceive a tree trunk visually at a distance sufficient to avoid it during migration. However, as also MüllerKraenner (1990) demonstrated in his species, this visual cue had to be greater than a certain minimum height: migrating colonies did not jam in front of a tree, but regularly against low obstacles like roots or branches lying on the ground. These could not be discerned at distance and were too high to overcome with a single leap.

Such an orientation facility does not indicate anything about visual orientation during climbing, when the animals head towards the next bark flake. There is no precise orientation needed, because it is enough when they head towards the dark bark in front of them. They need not to estimate any distance for a safe landing, because their body rolls on landing and this guarantees contact of their sticky vesicles with the substrate before they rebound or fall.

\section{Climbing onto trees}

On favourable days, almost the entire population can be found on the trees (Egli \& Kamke, 1996); the trunks appear to be covered with purple-grey paint, due to the springtails colonising the bark in densities up to 200 ind $/ \mathrm{cm}^{2}$ and being in body contact with each other.

When other springtail species jump during climbing, they shoot themselves of the surface and fall down to the ground. Even when coming into contact with the bark again, they will rebound. The situation is completely different in C. sigillata, which is able to jump successfully during climbing. The sticky vesicles avoid a fall, either by immediate contact with the bark or after a short roll. During all the experiments and observations in the climatic chamber as well as in the field, none of the springtails fell or rebounded, even when hitting a slippery vertical surface (glass or plastic, wet bark).

Christian (1979) demonstrated that the starting angle for Hypogastrura socialis was $77^{\circ}$. When jumping normally, $C$. sigillata performs leaps of similar height. However, from the tip of a bark flake or from a capillary in the laboratory, the animals always used a very low trajectory, i.e. for the shortest way out of the dead end. The starting angle in such cases was much lower than when taking off from a horizontal surface, this being attained by a stooped attitude of the body. For distances of 10 to $20 \mathrm{~mm}$, as they mostly occur in such situations, a maximum of 1 to 1.5 rotations can be calculated from the data given by Christian (1979) for H. socialis.

There is no choice of distance possible, but as mentioned above, the animals only need to head for the nearest vertical structure. In most cases they will hit the surface with their antennae first, but sometimes we also saw animals sticking with their anal vesicles. 
With this special jumping technique, $C$. sigillata is able to exploit the algal growth more easily on spruce trunks, which is of importance in increasing their cold hardiness, because they sequestrate antifreezes from the algae ( $\mathrm{J}$. Zettel, C. Suter \& S. Streich, in prep).

\section{Significance of vesicles}

Leinaas (1988) described anal vesicles from a number of poduromorph Collembola. In $H$. socialis, they have the form of fingerlike tubes, in other species, e.g. in C. sigillata, they are much shorter. Leinaas thought they were of significance for jumping, as he saw them being everted before takeoff; as we now know, they are everted just by the increased internal pressure when preparing a jump.

$\mathrm{He}$ also suggested an adaptive value of the anal vesicles, connected with pheromone release in readily jumping springtails; in such species the vesicles are much longer than in those without a distinct jumping behaviour (Leinaas, 1983).

As can be seen in the photographs published by Christian (1979), disturbed $H$. socialis do not show protruded anal vesicles (eversible antennal vesicles are not present in Hypogastrura species). His animals were stimulated to jump and therefore did not prepare to jump as seen in migrating animals; in fact the animals rebounded when hitting an obstacle in his experiments. Christian also mentioned that spontaneously jumping $H$. socialis often stuck to moist surfaces when jumping, but he offered no explanation for this phenomenon.

The ventral tube which is also protruded during a leap does not play a role as a sticking device in hypogastrurids, because it is too short to make contact with the surface. However, the ventral tube can be used as a climbing aid on slippery surfaces, a behaviour which is easily observed in culture vials. The situation during jumping can be quite different in sminthurids: Brackenbury (1990) described Sminthurus viridis using its long ventral tube to righten the body after a jump. Christian (1979) mentioned that in $S$. viridis the ventral tube is everted during a jump and with its moist surface might play an important role as a sticky landing aid within vegetation.

An adaptive value of protrusible vesicles can be postulated in migrating Collembola from the point of view of the more sophisticated landing technique; reducing in tumbling in regularly jumping species may be a mechanism to reduce time and energy spent during migration, especially when suitable environmental conditions prevail.

ACKNOWLEDGEMENTS. The present study was financially supported by the Swiss National Science Foundation and the WANDER-Stiftung zur Förderung der wissenschaftlichen Forschung an der Universität Bern. We thank W. Block for reading the manuscript, improving the English and for giving valuable comments. Further suggestions were given by an anonymous referee.

\section{REFERENCES}

Babenko A.B., Ciernova N.M., Potnpov M.B. \& Stebneva S.K. 1994: Collembola of Russia and Adjacent Countries; Familiy Hypogastruridae. Nauka, Moscow, 336 pp. (in Russian).

BAUER T. \& VÖLIENkLE W. 1976: Hochfrequente Filmaufnahmen als Hilfsmittel bei der Analyse von Angriffs- und Fluchtverhalten in einer Räuber-Beute-Beziehung unter Bodentieren (Collembolenfang visuell jagender Carabiden). Wiss. Film, Wien, pp. 4-11.

BRACKLNBURY J. 1990: A novel method of self-righting in the springtail Sminthurus viridis (Insecta: Collembola). J. Zool. 222: $117-119$.

Brackenbury J. \& Hunt H. 1993: Jumping in springtails: mechanisms and dynamics. J. Zool. 229: 217-236.

ChristinN E. 1978: The jump of the springtails. Natumissenschaften 65: 495.

Cirrisitinn E. 1979: Der Sprung der Collembolen. Zool. Jb. Physiol. 83: 457-490.

EGGER B. 1998: Richtungshüpfen und Hüpffrequenz von Ceratophysella sigillata (Collembola, Hypogastruridae). Unpubl. report, Zool. Inst. Univ. Bern, $10 \mathrm{pp}$.

EgLi B. \& Kamke M.C. 1996: Alterszusammensetzung und Koloniegrösse bei Ceratophysella sigillata. Unpubl. report, Zool. Inst. Univ. Bern, 13 pp.

HÅvar S. 1995: Long distance, directional migration on snow in a forest Collembole, Hypogastrura socialis (Uzel). Acta Zool. Fenn. 196: 200-205.

LLiNAMS H.P. 1981a: Cyclomorphosis in the furca of the winter active Collembola Hypogastrura socialis (Uzel). Entomol. Scand. 12: 35-38.

LFINAAS H.P. 1981 b: Cyclomorphosis in Hypogastrura lapponica (Axelson, 1902) [=H. frigida (Axelson, 1905) syn. nov.] (Collembola, Poduridae). Morphological adaptations and selection for winter dispersal. Z. Zool. Syst. Evol. Forsch. 19: 278--285.

LEINAAS H.P. 1983: Synchronized moulting controlled by communication in group-living Collembola. Science (Washington) 219: 193-195.

Leinı s H.P. 1988: Anal sacks - an unknown organ in Poduromorpha (Collembola). Zool. Scripta 17: 227-284.

LYlord W.H. 1975: Overland migration of Collembola (Hypogastrura nivicola Fitch) colonies. Am. Midl. Nat. 94: 205-209.

Müller-Kratannir S. 1990: Entwicklung, Ökologie und Verhalten von Entomobrya corticalis (Nicolet 1841) im Vergleich mit drei anderen epigäischen Collembolen-Arten. Diploma thesis, Univ. Bayreuth, 96 pp.

PAirss A. 1964: Apterygota. In Brohmer P. (ed.): Tierwelt Mitteleuropas. Quelle \& Meyer, Leipzig, 405 pp.

RUHFUS B. \& ZINK1.FR D. 1995: Investigations on the sources utilized for the energy supply fuelling the jump of springtails. J. Insect Physiol, 41: 297-301.

Sutter C. 1993: Beiträge zur Nahrungsökologie von Ceratophysella sigillata (Collembola: Hypogastruridae). Diploma thesis, Zool. Inst. Univ. Bern, 31 pp.

ZETTEL J. \& ZEI"TLL U. 1994a: Development, phenology and surface activity of Ceratophysella sigillata (Uzel) (Collembola: Hypogastruridae). Acta Zool. Fenn. 195: 150-153.

ZETTEL U. \& ZITTLL J. 1994b: Seasonal and reproductional polymorphism in Ceratophysella sigillata (Uzel) (Collembola: $\mathrm{Hy}$ pogastruridae). Acta Zool. Fenn. 195: 154-156.

Received March 16, 1999; accepted July 17, 1999 